\title{
Angiographic Analysis of the Anatomical Variants in Genicular Artery Embolization
}

\author{
Ari Isaacson ${ }^{4}$ \\ ${ }^{1}$ Vascular Interventional Partners - NOVA, Falls Church, Virginia, \\ United States \\ ${ }^{2}$ Fauquier Hospital, Warrenton, Virginia, United States \\ ${ }^{3}$ NYU Winthrop Hospital, Department of Surgery, St. Mineola, \\ New York, United States \\ ${ }^{4}$ University of North Carolina at Chapel Hill School of Medicine, \\ Chapel Hill, North Carolina, United States
}

Sandeep Bagla ${ }^{1}$ Rachel Piechowiak ${ }^{2}$ Abin Sajan ${ }^{3, \odot ~ J u l i e ~ O r l a n d o ~}{ }^{2}$

Diego A. Hipolito Canario ${ }^{4}, \odot$

\begin{abstract}
Address for correspondence Sandeep Bagla, MD, Vascular Interventional Partners - NOVA, 2755 Hartland Road, Falls Church, VA 22043, United States (e-mail: sandeep.bagla@gmail.com).
\end{abstract}

J Clin Interv Radiol ISVIR 2021;6:18-22.

Purpose Genicular artery embolization (GAE) has been proposed as a novel technique to treat painful synovitis related to osteoarthritis. An in-depth understanding of the genicular arterial anatomy is crucial to achieve technical success and avoid nontarget-related complications. Given the lack of previous angiographic description, the present study analyzes genicular arterial anatomy and proposes an angiographic classification system.

Materials and Methods Angiographic findings from 41 GAEs performed during two US clinical trials from January 2017 to July 2019 were reviewed to analyze the anatomical details of the following vessels: descending genicular artery (DGA), medial superior genicular artery (MSGA), medial inferior genicular artery (MIGA), lateral superior genicular artery (LSGA), lateral inferior genicular artery (LIGA), and anterior tibial recurrent artery (ATRA). The diameter, angle of origin, and anastomotic pathways were recorded for each vessel. The branching patterns were classified as: medially, M1 (3/3 arteries present) vs M2 (2/3 arteries present); and laterally, L1 (3/3 arteries present) vs $L 2$ ( $2 / 3$ arteries present).

Results A total of 91 genicular arteries were embolized: DGA (26.4\%), MIGA (23.1\%), MSGA (22.0\%), LIGA (14.3\%), and LSGA/ATRA (14.3\%). The branching patterns were: medially $=$ M1, 74.4\% $(n=29), M 2,25.6 \%(n=10)$; and laterally $=\mathrm{L} 1,94.9 \%(n=37), L 2$, $5.1 \%(n=2)$. A common origin for MSGA and LSGA was noted in 11 patients $(28.2 \%)$. A direct DGA origin from the popliteal artery was reported in three patients $(7.7 \%, n=3)$. Conclusions A thorough understanding of the geniculate arterial anatomy is important for maximizing postprocedural pain reduction while minimizing complications, procedure time, and radiation exposure during GAE. published online April 29, 2021
DOI https://doi.org/

$10.1055 / \mathrm{s}-0041-1729464$ ISSN 2457-0214 (c) 2021. Indian Society of Vascular and Interventional Radiology. This is an open access article published by Thieme under the terms of the Creative Commons Attribution-NonDerivative-NonCommercial-License, permitting copying and reproduction so long as the original work is given appropriate credit. Contents may not be used for commercial purposes, or adapted, remixed, transformed or built upon. (https://creativecommons.org/licenses/by-nc-nd/4.0/).

Thieme Medical and Scientific Publishers Pvt. Ltd. A-12, 2nd Floor, Sector 2, Noida-201301 UP, India 


\section{Introduction}

Genicular artery embolization (GAE) is a novel, minimally invasive therapy for patients with mild to moderate osteoarthritic (OA) knee pain. ${ }^{1}$ Initially described for hemarthrosis, ${ }^{2,3}$ GAE for OA pain has shown promising 4-year follow-up results from its initial investigations. ${ }^{4-6}$ Because of the encouraging initial results, further prospective and randomized-controlled trials are currently underway in the United States, Japan, and the United Kingdom.

In the majority of patients with knee OA, the articular cartilage breakdown is associated with chronic synovitis. ${ }^{7}$ This inflammatory process results in the formation of new blood vessels and subsequent recruitment of new sensory nerve fibers within the synovium, resulting in chronic knee pain. ${ }^{8,9}$ The goals of embolizing the neovascularity are twofold: cause ischemic neurolysis of the synovial sensory nerves and disrupt the inflammatory positive feedback cycle, ultimately resulting in decreased pain and disability. ${ }^{6,10-13}$

The success of GAE, as well as the prevention of nontarget embolization, is related to an in-depth understanding of the genicular arterial anatomy. Several studies ${ }^{14-16}$ have addressed this topic using cadavers, but an angiographic analysis is yet to be reported. The present study describes genicular angiographic findings, proposes an angiographic classification, and discusses implications of genicular arterial variations for the GAE procedure.

\section{Materials and Methods}

The study was approved by the local institutional review board and all study activities followed the Health Insurance Portability and Accountability Act regulations.

Angiographic findings from 41 GAE procedures performed in 40 subjects to treat OA pain from January 2017 to July 2019 were reviewed. Angiograms from two procedures were unable to be analyzed due to technical reasons and were excluded from the study for a total of 38 patients and 39 procedures. At baseline, all patients had clinical (pain at least 5 on a scale of 10) and radiographic evidence of knee osteoarthritis (Kellgren-Lawrence Grade 1-3 severity). Response to therapy was assessed with the Western Ontario McMaster Universities Osteoarthritis Index and a Visual Analog Scale for pain. Additionally, incidence and severity of adverse events were evaluated at baseline, 1-, 3-, and 6-month follow-up. Clinical results from one of the two clinical studies has been published previously. ${ }^{17}$

Procedures were performed according to previously described techniques.5,6,17 Under moderate sedation, arterial access was obtained through the contralateral femoral artery with a $6 \mathrm{~F}$ sheath. Subsequent lower-extremity digital subtraction angiography was performed from the distal femoral artery to capture the complete genicular arterial anatomy between the origins of the descending genicular artery (DGA) and anterior tibial recurrent artery (ATRA). A 2.4F microcatheter (Terumo, Princeton NJ, or Boston Scientific, Natick, Massachusetts) was used to catheterize specific genicular arteries and deliver 75-300 um spherical particles
(Embozene: Boston Scientific, Natick, Massachusetts; or Optisphere: Medtronic, Minneapolis, MN) to hypervascular regions consistent with areas of pain.

Three interventional radiologists with experience (7-9 years) performing embolizations analyzed all arteriograms and studied the following vessels: DGA, medial superior genicular artery (MSGA), medial inferior genicular artery (MIGA), lateral superior genicular artery (LSGA), lateral inferior genicular artery (LIGA), and ATRA. The presence of the arteries, diameter within $1 \mathrm{~cm}$ of their origin, branching pattern, angle of origin to the proximal parent vessel, anastomotic network, and distance from the origin of the DGA were recorded for each vessel. Additional measurements included popliteal diameter $1 \mathrm{~cm}$ below the DGA origin and radiation dosage. The average fluoroscopy time was compared between patients with all vessels present (M1 or L1) and patients with at least one vessel missing (M2 or L2) to evaluate the effects of anatomical variations on overall procedural time and radiation dose.

The branching patterns were classified into following subtypes:

1. Medially:

- M1 (presence of all three medial branches: DGA, MSGA, MIGA).

- M2 (presence of two of the three medial branches: either DGA and MSGA or DGA and MIGA).

\section{Laterally:}

- L1 (presence of all three lateral branches: LSGA, LIGA, ATRA).

- L2 (presence of two of the three lateral branches: either ATRA and LSGA or ATRA and LIGA).

\section{Results}

- Fig. 1(A-F) identifies the six main arteries being studied. Of the 108 total arteries selected, 91 were treated: DGA (24/91, 26.4\%), MIGA (21/91, 23.1\%), MSGA (20/91, 22.0\%), LIGA (13/91, 14.3\%), and LSGA/ATRA (13/91, 14.3\%). The difference in selected vs embolized arteries refers to catheterized

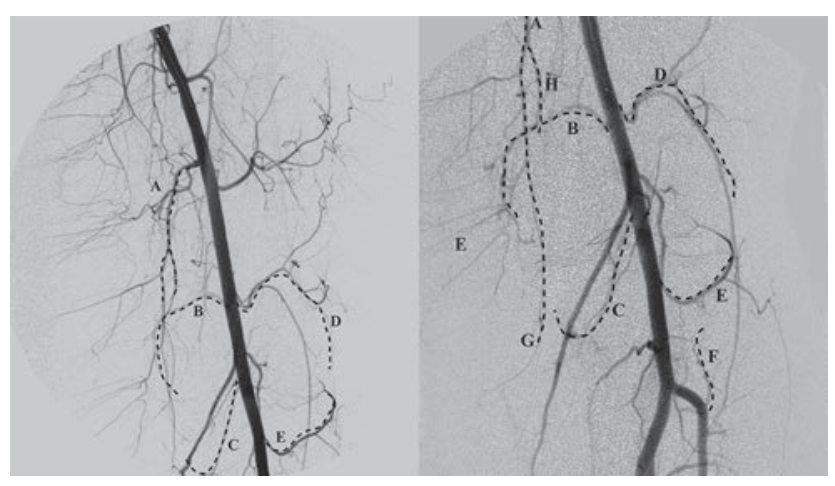

Fig. 1 Overview of the genicular arteries: (A) descending genicular artery (DGA); (B) medial superior genicular artery; (C) medial inferior genicular artery; (D) lateral superior genicular artery; (E) lateral inferior genicular artery; (F) anterior tibial recurrent artery; (G) saphenous branch of DGA; $(\mathbf{H})$ musculoarticular branch of DGA. 
vessels in the region of pain that were negative for hypervascularity and therefore did not receive treatment. The following branching patterns were observed-medially: $\mathrm{M} 1=74.4 \%$ ( $n=29 / 39), \mathrm{M} 2=25.6 \%(n=10 / 39)$; laterally: $\mathrm{L} 1=94.9 \%$ $(n=37 / 39), \mathrm{L} 2=5.1 \%(n=2 / 39)$. The M1 and L1 branching pattern was seen in 27 procedures and the M2 or L2 branching pattern was seen in 12 procedures. A common origin for MSGA and LSGA was noted in 11 out of 39 procedures (28.2\%). Additional analysis of the DGA branching pattern revealed direct DGA origin from the popliteal artery in 3 out of 39 procedures $(7.7 \%, n=3)$. The procedure was performed bilaterally on one patient and right vs left knee analysis revealed a M1L1 branching pattern on both sides, but a common origin for the MSGA and LSGA was noted on the right side.

- Table 1 summarizes the frequency, diameter, angle of origin, and distance from DGA for all six arteries. Anastomotic supply to another genicular artery in selected vessels was recorded $(26.4 \%, 24 / 91)$. Anastomotic relationships were noted between DGA and MSGA/LSGA, MIGA and LIGA, and LSGA and LIGA/ATRA. The average popliteal diameter was $7.55 \pm 1.54 \mathrm{~mm}$ and the average administered reference air kerma level was $128.31 \pm 106.21$. No significant difference was noted when comparing mean fluoroscopy time, in minutes, of M1 or L1 $(n=27)$ vs M2 or L2 $(n=12)$ : $27.47 \pm 13.8$ vs $20.47 \pm 5.56 ; p=0.10$.

\section{Discussion}

\section{Classification}

Knee pain in osteoarthritis can generally be lateralized to either the medial or lateral aspect of the knee. The first step in GAE involves mapping the vasculature associated with the corresponding painful region of the knee. Cadaveric studies have previously reported variations in genicular arterial anatomy and proposed two different classification patterns. ${ }^{18,19}$ The classification types mainly involve differences in the middle genicular artery (MGA) branching pattern and its common origin with other genicular arteries. The MGA was not treated in the present study given its limited perfusion of the knee. The purpose of the current classification system is to create a clinically oriented model that could be used for consistent reporting in future GAE studies. Additionally, the classification system could help predict the overall procedural time and give information about the complexity of anastomotic networks.

\section{Medial Compartment}

The medial compartment of the knee is perfused by the DGA, MSGA, and MIGA. The DGA originates from the distal superficial femoral artery (SFA) and has an inverted "Y" appearance. It divides into a straight medial saphenous branch, which courses superficially, and a more torturous lateral musculoarticular branch, which courses deeper in the knee. The DGA was present in all patients in this analysis. Anatomical studies have reported variations in DGA anatomy with isolated origins of the DGA branches from the distal SFA. ${ }^{20}$ Although similar variations were not present in the current study, the DGA originated in three patients from the above-knee popliteal artery instead of the distal SFA. The MSGA was the most commonly absent of the medial genicular arteries (present $84.6 \%$ of the time) and had the smallest mean diameter $(1.2 \mathrm{~mm})$. In some patients, the MSGA was less than $1 \mathrm{~mm}$ and could not be catheterized. It is important to adequately evaluate for collateral pathways of perfusion in patients with a very small or absent MSGA, performing high-quality angiography of both the MIGA and DGA.

The MIGA originates from the popliteal artery near the joint space and descends along the upper margin of the popliteus, before coursing anteriorly and superiorly creating the angiographic " $V$ " around the medial tibial metaphysis. The MIGA was absent in four patients and should be distinguished from the adjacent sural arteries, which follow a straight downward course toward the musculature.

Table 1 Summary of genicular artery measurements

\begin{tabular}{|c|c|c|c|c|}
\hline Artery & Frequency & Diameter & Angle $(<90)$ & Distance \\
\hline \multirow[t]{2}{*}{ DGA } & \multirow{2}{*}{$\begin{array}{l}39 / 39 \\
100.0 \%\end{array}$} & 2.4 & \multirow[t]{2}{*}{$5.4 \%$} & NA \\
\hline & & 0.8 & & NA \\
\hline \multirow[t]{2}{*}{ MSGA } & \multirow{2}{*}{$\begin{array}{l}33 / 39 \\
84.6 \%\end{array}$} & 1.2 & \multirow[t]{2}{*}{$97.1 \%$} & 10.6 \\
\hline & & 0.5 & & 3.6 \\
\hline \multirow[t]{2}{*}{ MIGA } & \multirow{2}{*}{$\begin{array}{l}35 / 39 \\
89.7 \%\end{array}$} & 1.6 & \multirow[t]{2}{*}{$8.8 \%$} & 17.3 \\
\hline & & 0.5 & & 4.4 \\
\hline \multirow[t]{2}{*}{ LSGA } & \multirow{2}{*}{$\begin{array}{l}38 / 39 \\
97.4 \%\end{array}$} & 1.5 & \multirow[t]{2}{*}{$94.6 \%$} & 11.6 \\
\hline & & 0.4 & & 4.0 \\
\hline \multirow[t]{2}{*}{ LIGA } & \multirow{2}{*}{$\begin{array}{l}38 / 39 \\
97.4 \%\end{array}$} & 1.5 & \multirow[t]{2}{*}{$71.4 \%$} & 18.7 \\
\hline & & 0.4 & & 4.9 \\
\hline \multirow[t]{2}{*}{ ATRA } & \multirow{2}{*}{$\begin{array}{l}39 / 39 \\
100.0 \%\end{array}$} & 1.6 & \multirow[t]{2}{*}{$100.0 \%$} & 25.7 \\
\hline & & 0.5 & & 4.6 \\
\hline
\end{tabular}

Abbreviations: ATRA, anterior tibial recurrent artery; DGA, descending genicular artery; LIGA, lateral inferior genicular artery; LSGA, lateral superior genicular artery; MIGA, medial inferior genicular artery; MSGA, medial superior genicular artery, NA, not available.

Note: All distance and diameter measures $(\mathrm{mm})$ are summarized as mean values along with the respective standard deviations. The angle of the selected vessel was compared to the proximal parent artery (e.g., popliteal artery). 


\section{Lateral Compartment}

The lateral compartment is perfused by the LSGA, LIGA, and ATRA. Compared to the medial compartment, less variation was noted in the lateral vasculature. The LSGA originates from the popliteal artery at the level of the lateral femoral condyle and splits into the superficial and deep branches. The LIGA originates around the joint space and courses laterally above the fibular head before wrapping around the lateral tibial condyle, creating a "J" appearance. The LSGA and LIGA were rarely absent but a common origin of the MSGA and LSGA was noted in 11 patients (28.2\%). Although the MGA was not analyzed in the present study, anatomical studies have previously reported common branching between MSGA, LSGA, and MGA. ${ }^{21,22}$ This common trunk may make catheterization of each target vessel more challenging, especially given its frequent acute angle of origin.

The ATRA is the most inferior branch supplying the knee and originates from the anterior tibial artery just proximal to its origin. The ATRA was present in all patients with an acute angle of origin relative to the anterior tibial artery.

\section{Anastomotic Networks}

Anastomoses between genicular arteries were often observed when injecting a specific genicular artery during angiography $(26.4 \%, 24 / 91)$. Observation of anastomoses was highest in cases with variant anatomy (M2 and L2). The most common anastomosis was noted between the musculoarticular branch of the DGA and the MSGA/LSGA (-Fig. 2). This relationship can be explained given that the MSGA is the most commonly absent vessel and had the smallest diameter of all six vessels. Similar pathways were observed between other genicular branches, highlighting the need to explore all three vessels on the symptomatic side for anastomosis and variant anatomy. Although selecting all vessels can increase the

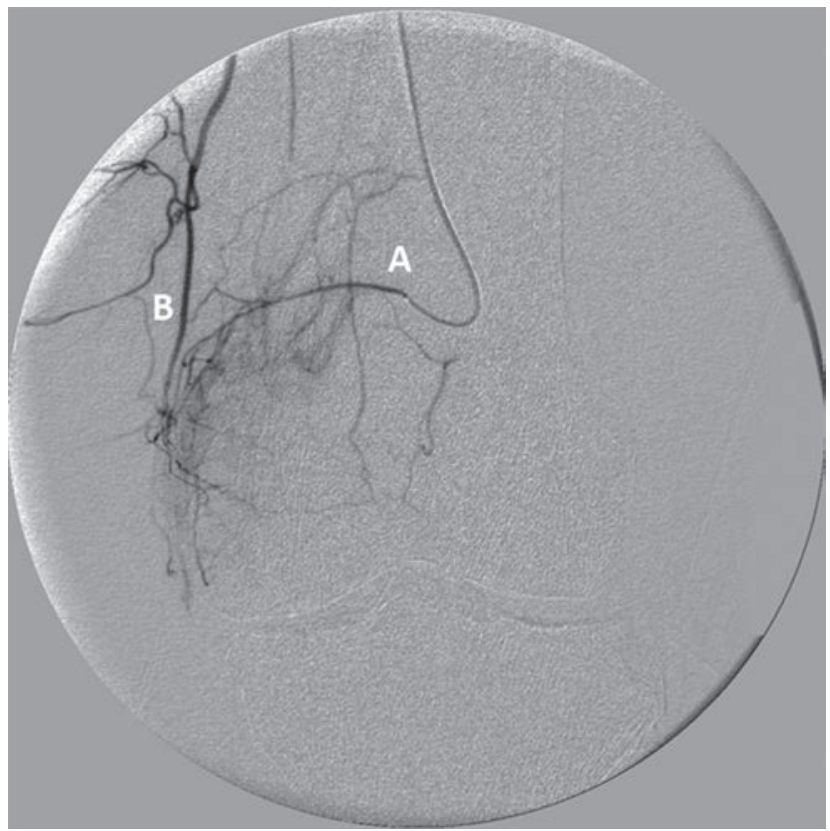

Fig. 2 The medial superior genicular artery (MSGA) (A) is selected and anastomoses between the MSGA and the musculoarticular branch of the descending genicular artery (B) is visualized. fluoroscopy time, significant difference in fluoroscopy time was not observed while comparing M1/L1 vs M2/L2.

\section{Challenges}

One of the major challenges in GAE is correctly identifying and navigating the vascular anatomy, particularly the angle of origin. The angle of origin can prolong catheterization time, fluoroscopy time, and has implications for proper catheter selection. The angle of origin in most vessels was obtuse relative to the proximal popliteal artery, providing an easier angle to catheterize, and thereby allowing the use of nonshaped microcatheters. The MSGA/LSGA/ATRA are difficult arteries to catheterize given the acute angle of

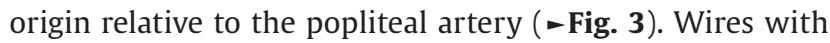
longer length and flexible tips may not provide enough support to advance the microcatheter, and preshaped microcatheters can offer an advantage. The operators in this study used a variety of wire and microcatheter combinations at their discretion.

Another major challenge to GAE is preventing nontarget embolization. Cutaneous arteries may be confused for genicular synovial branches leading to multiple side-effects. Cutaneous arteries appear perpendicular to the main genicular arteries extending to the cutaneous surface, and embolizing proximal to these vessels may lead to cutaneous ischemia. Although most often reported as subclinical and self-resolving, the possibility of ulceration is present. The presence of a common origin, especially of MSGA/LSGA, is an important consideration, as reflux could lead to nontarget embolization to the asymptomatic side. Similarly, collateral networks can cross the joint space and anastomose with ipsilateral and contralateral vessels. Recognizing the typical anatomy and anticipating for variants are vital for best patient outcomes and minimizing risk.

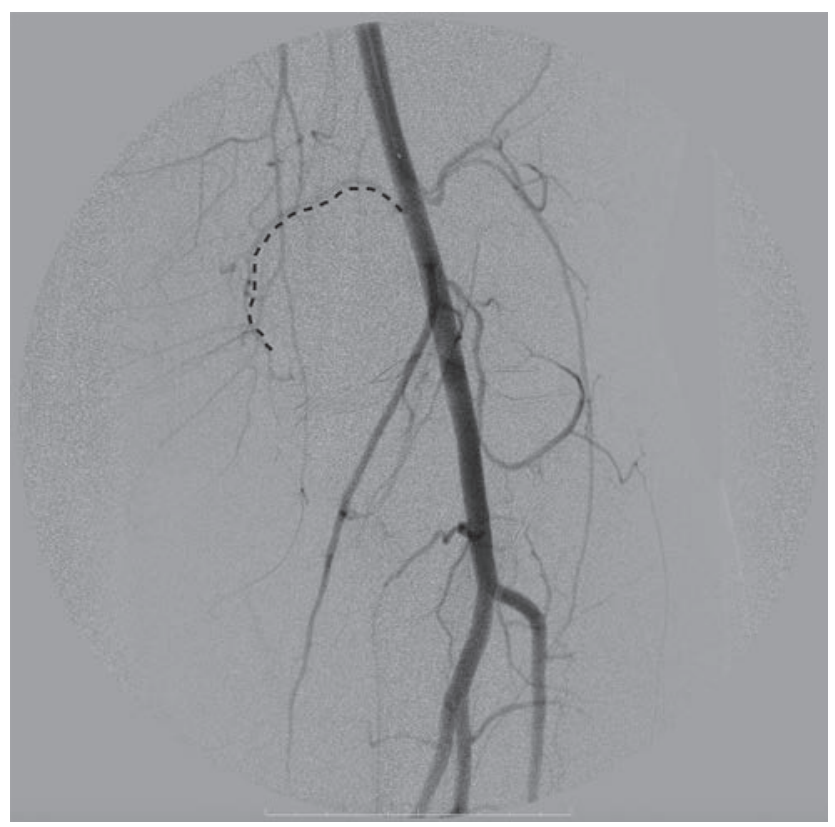

Fig. 3 The acute angle of origin of the medial superior genicular artery relative to the proximal popliteal artery is demonstrated. 


\section{Conclusion}

The knee joint has a complex vascular network with extensive anastomotic networks and anatomical variations. During GAE, a thorough understanding of the genicular artery anatomy is important for maximizing clinical outcomes for pain reduction while minimizing complications, procedure time, and radiation exposure. As experience increases with GAE, further correlation with clinical and magnetic resonance imaging findings will be useful in preoperative planning for embolization.

\section{Note}

This paper was accepted/presented at the Society of Interventional Radiology Annual Meeting, 2020.

\section{Conflict of Interest}

Dr. Isaacson reports other from Terumo, other from ABK Biomedical, other from CrannMed, outside the submitted work. Dr. Bagla reports other from Boston Scientific, other from Varian Medical Systems, other from Medtronic, other from Embolx, other from IMBiotechnologies, other from Phillips Medical System, outside the submitted work. All other authors reported no conflict of interest.

\section{References}

1 Goldman DT, Piechowiak R, Nissman D, Bagla S, Isaacson A. Current concepts and future directions of minimally invasive treatment for knee pain. Curr Rheumatol Rep 2018;20(9):54

2 Bagla S, Rholl KS, van Breda A, Sterling KM, van Breda A. Geniculate artery embolization in the management of spontaneous recurrent hemarthrosis of the knee: case series. J Vasc Interv Radiol 2013;24(3):439-442

3 Yoo JH, Oh HC, Park SH, Lee S, Lee Y, Kim SH. Treatment of recurrent hemarthrosis after total knee arthroplasty. Knee Surg Relat Res 2018;30(2):147-152

4 Lee SH, Hwang JH, Kim DH, et al. Clinical outcomes of transcatheter arterial embolisation for chronic knee pain: mild-to-moderate versus severe knee osteoarthritis. Cardiovasc Intervent Radiol 2019;42(11):1530-1536

5 Okuno Y, Korchi AM, Shinjo T, Kato S. Transcatheter arterial embolization as a treatment for medial knee pain in patients with mild to moderate osteoarthritis. Cardiovasc Intervent Radiol 2015;38(2):336-343

6 Okuno Y, Korchi AM, Shinjo T, Kato S, Kaneko T. Midterm clinical outcomes and MR imaging changes after transcatheter arterial embolization as a treatment for mild to moderate radiographic knee osteoarthritis resistant to conservative treatment. J Vasc Interv Radiol 2017;28(7):995-1002
7 Sokolove J, Lepus CM. Role of inflammation in the pathogenesis of osteoarthritis: latest findings and interpretations. Ther Adv Musculoskelet Dis 2013;5(2):77-94

8 Costa C, Incio J, Soares R. Angiogenesis and chronic inflammation: cause or consequence? Angiogenesis 2007;10(3):149-166

9 Grässel S, Muschter D. Peripheral nerve fibers and their neurotransmitters in osteoarthritis pathology. Int J Mol Sci 2017;18(5):E931

10 Ashraf S, Mapp PI, Walsh DA. Contributions of angiogenesis to inflammation, joint damage, and pain in a rat model of osteoarthritis. Arthritis Rheum 2011;63(9):2700-2710

11 Bonnet CS, Walsh DA. Osteoarthritis, angiogenesis and inflammation. Rheumatology (Oxford) 2005;44(1):7-16

12 Mapp PI, Walsh DA. Mechanisms and targets of angiogenesis and nerve growth in osteoarthritis. Nat Rev Rheumatol 2012;8(7):390-398

13 Okuno Y, Oguro S, Iwamoto W, Miyamoto T, Ikegami H, Matsumura N. Short-term results of transcatheter arterial embolization for abnormal neovessels in patients with adhesive capsulitis: a pilot study. J Shoulder Elbow Surg 2014;23(9):e199-e206

14 Salaria H, Atkinson R. Anatomic study of the middle genicular artery. J Orthop Surg (Hong Kong) 2008;16(1):47-49

15 Sananpanich K, Atthakomol P, Luevitoonvechkij S, Kraisarin J. Anatomical variations of the saphenous and descending genicular artery perforators: cadaveric study and clinical implications for vascular flaps. Plast Reconstr Surg 2013;131(3):363e-372e

16 Yasar E, Kesikburun S, Kılıç C, Güzelküçük Ü, Yazar F, Tan AK. Accuracy of ultrasound-guided genicular nerve block: a cadaveric study. Pain Physician 2015;18(5):e899-e904

17 Bagla S, Piechowiak R, Hartman T, et al. Genicular artery embolization for the treatment of knee pain secondary to osteoarthritis. J Vasc Interv Radiol 2020;31(7):1096-1102

18 Shahid S, Saghir N, Cawley O, Saujani S. A cadaveric study of the branching pattern and diameter of the genicular arteries: a focus on the middle genicular artery. J Knee Surg 2015;28(5):417-424

19 Yang K, Park JH, Jung S, et al. Topography of the middle genicular artery is associated with the superior and inferior genicular arteries. Int J Morphol 2017;35:913-918

20 Ziegler T, Kamolz LP, Vasilyeva A, Schintler M, Neuwirth M, Parvizi D. Descending genicular artery. Branching patterns and measuring parameters: a systematic review and meta-analysis of several anatomical studies. J Plast Reconstr Aesthet Surg 2018;71(7):967-975

21 Bettaiah A, Venkat S, Saraswathi G. A study of variations in the branching pattern of popliteal artery and its clinical perspective. Int J Res Med Sci 2016;4(8):3584-3589

22 Singla R, Kaushal S, Chabbra U. Popliteal artery branching pattern: a cadaveric study. Eur J Anat 2012;16:157-162 